\title{
Microchannel Molecular Communication with Nanoscale Carriers: Brownian Motion versus Active Transport
}

\author{
Andrew W. Eckford*, Member, IEEE, Nariman Farsad, Student Member, IEEE, \\ Satoshi Hiyama, Member, IEEE and Yuki Moritani
}

\begin{abstract}
In molecular communication, information is encoded and transmitted as a pattern of molecules or other very small information carriers (in this paper, vesicles are used). Nanoscale techniques, such as molecular motors or Brownian motion, are used to convey the vesicles from the transmitter to the receiver, where the transmitted message is deciphered. In this paper, the microchannel environment is considered, and the achievable information rates are compared between the use of Brownian motion and molecular motors, which are evaluated through simulation. Communication is viewed as a mass transfer problem, where messages are sent by transporting a number of vesicles from transmitter to receiver. Results are provided which suggest that active transport is best when the available number of vesicles is small, and Brownian motion is best when the number of vesicles is large.
\end{abstract}

\section{INTRODUCTION}

Molecular communication [1] is a new and biologicallyinspired communication technique which uses nanoscale properties of materials. Using molecular communication, information is conveyed by encoding messages into the timing or identities of molecules, which are released by a transmitter and propagate to a receiver.

There is an emerging body of literature examining molecular communication from an engineering perspective. One aspect of this literature explores the various nanotechnological and biological techniques that can be exploited in order to create a communication system, such as calcium gap junctions in cells [2], [3] and molecular motors propagating along along cytoskeletal filaments [4], [5]. (The reader is directed to [6] for a thorough survey of molecular communication techniques.) Another aspect of the literature explores molecular communication as a communication system, i.e., from a communicationtheoretic and information-theoretic perspective. Notable works in this direction include a general formulation of molecular communication as a timing channel under Brownian motion [7], [8], and an analysis of information transfer rates using molecular motors [9], [10]. Of the existing literature, these information-theoretic papers are most closely related, though

* Corresponding author.

Andrew W. Eckford and Nariman Farsad are with the Department of Computer Science and Engineering, York University, 4700 Keele Street, Toronto, ON, Canada M3J 1P3. Emails: aeckford@yorku.ca, nariman@cse.yorku.ca

Satoshi Hiyama and Yuki Moritani are with Research Laboratories, NTT DOCOMO Inc., Yokosuka, Kanagawa, Japan. Emails: \{hiyama, moritani\}@nttdocomo.co.jp as we explain later, we propose a novel way of viewing the information transfer problem.

One important practical domain for molecular communication is in the microchannel environment, assuming the labon-a-chip devices without using the microfluidic flow, where chemical processes are carried out in very small engineered spaces [11]. In these systems, information may need to be transmitted from one or more reaction sites to a data fusion center, where a decision is made (e.g., deciding on the presence or absence of pathogens). Though most conventional communication systems are electrical or electromagnetic in nature, it is generally inconvenient to combine electrical and chemical components on the same device; thus, molecular communication is an obvious solution.

The fundamental components of the microchannel system have nanoscale dimensions, and it is these components that we study in this paper. We consider a microchannel molecular communication system consisting of nanoscale information carriers (i.e., molecules or vesicles). Further, this study is motivated by the existence of two possible nanoscale mass transport systems: Brownian motion or active transport (in our scheme, "active transport" refers to the transportation of information-bearing vesicles using gliding microtubules driven by immobilized kinesin molecular motors [12]). It is unclear which method is most efficient to use in order to construct a molecular communication system, or whether each method is most appropriate in different circumstances. Thus, our main contribution is to comparatively evaluate the achievable information rates using both molecular motors and simple Brownian motion. To this end, we produce a simulation environment of the nanoscale components in a microchannel system, and generate information transmission models based on these simulations; finally, the models are used to analyze the system's information-theoretic properties. To our knowledge, our paper provides the first direct comparison between Brownian motion and active transport in an information transmission problem.

\section{Simulation ENVIRONMENT AND MODELS}

Our setup, shown in Figure 1, is similar to that given in [13]. We use a rectangular propagation environment (with rounded corners), consisting of a loading zone and an unloading zone. Regardless of the propagation model, message-bearing vesicles 


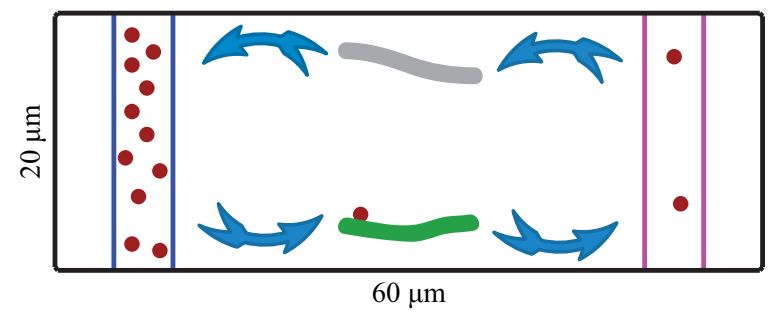

Fig. 1. Depiction of the simulation environment. In this figure, dots represent vesicles, and filaments represent microtubules; note that the microtuble at bottom has loaded a vesicle, whereas the microtubule at top has no vesicle. The loading zone is on the left, and the unloading zone is on the right. Microtubules and vesicles are not to scale.

originate at the loading zone, and propagate until they arrive at the unloading zone. In this figure, it is important to note that the microtubules and vesicles are not to scale.

We perform a two-dimensional discrete-time simulation of particle motion (whether the particle is a vesicle or a microtubule). Given some initial position $\left(x_{0}, y_{0}\right)$ at time $t=0$, for any integer $i>0$, the motion of the particle is given by the sequence of coordinates $\left(x_{i}, y_{i}\right)$. Each coordinate $\left(x_{i}, y_{i}\right)$ represents the position of the particle at the end of time $t=i \Delta t$, where

$$
\begin{aligned}
x_{i} & =x_{i-1}+\Delta r \cos \theta_{i}, \\
y_{i} & =y_{i-1}+\Delta r \sin \theta_{i} .
\end{aligned}
$$

The values of $\Delta r$ and $\theta_{i}$ are dependent on the propagation model in use. We consider two models in this paper: Brownian motion, where the particle is a vesicle; and active transport, where the particle is a microtubule. Two-dimensional simulations are appropriate for microtubule propagation along molecular motors (since the motors do not allow vertical propagation), and we also use them for Brownian motion for the sake of simplicity.

Mathematically, it is well known that Brownian motion can be described by stochastic differential equations, and properties of the motion (such as first arrival time distributions) can be derived from solutions of these equations. However, in confined spaces, such as microchannels, solutions are generally not available in closed form. Furthermore, analytical solutions are generally unknown for active transport. Thus, following [14], we performing Monte Carlo simulations on particles in order to obtain the needed properties of the motion.

\section{A. Brownian motion}

Brownian motion refers to the random motion of a particle as it collides with other molecules in its vicinity. Over each time interval of $\Delta t$, the molecule's displacement $\Delta r$ is given by

$$
\Delta r=\sqrt{4 D \Delta t}
$$

where $D$ is the free diffusion coefficient. For a given molecule and fluid propagation environment, $D$ is given by

$$
D=\frac{k_{B} T}{6 \pi \eta R_{H}},
$$

where $k_{B}=1.38 \cdot 10^{-23} \mathrm{~J} / \mathrm{K}$ is the Boltzman constant, $T$ is the temperature (in $\mathrm{K}$ ), $\eta$ is the dynamic viscosity of the fluid, and $R_{H}$ is the hydraulic radius of the molecule. We assume that $D$ is the same throughout the medium, and that collisions with the boundaries are elastic. In [14], values of $D$ ranging from $1-10 \mu \mathrm{m}^{2} / \mathrm{s}$ were considered realistic for signalling molecules. Further, $\theta_{i}$ is an independent, identically distributed (iid) random variable for all $i$, uniformly distributed on $[0,2 \pi)$.

\section{B. Molecular motors}

As in [13], we assume that the microchannel is lined with static kinesin motors, and that these motors cause microtubules to propagate along their surface. The motion of the microtubule is largely regular, although the effects of Brownian motion cause random fluctuations. We use Monte Carlo simulation to obtain the needed properties of the motion, using a scheme from [15]. In this case, the step size $\Delta r$ at each step is an iid Gaussian random variable with mean and variance

$$
\begin{aligned}
E[\Delta r] & =v_{\mathrm{avg}} \Delta t \\
\operatorname{Var}[\Delta r] & =2 D \Delta t
\end{aligned}
$$

where $v_{\text {avg }}$ is the average velocity of the microtubule, and $D$ is the microtubule's diffusion coefficient. The angle $\theta_{i}$ is no longer independent from step to step: instead, for some stepto-step angular change $\Delta \theta$, we have that

$$
\theta_{i}=\Delta \theta+\theta_{i-1} .
$$

Now, for each step, $\Delta \theta$ is an iid Gaussian-distributed random variable with mean and variance

$$
\begin{aligned}
E[\Delta \theta] & =0, \\
\operatorname{Var}[\Delta \theta] & =\frac{v_{\mathrm{avg}} \Delta t}{L_{p}},
\end{aligned}
$$

where $L_{p}$ is the persistence length of the microtubule's trajectory. In [15], these values were given as $v_{\text {avg }}=0.85 \mu \mathrm{m} / \mathrm{s}$, $D=2.0 \cdot 10^{-3} \mu \mathrm{m}^{2} / \mathrm{s}$, and $L_{p}=111 \mu \mathrm{m}$. Following [15], in case of a collision with a boundary, we assume that the microtubule does not reflect off the boundary, as in an elastic collision, but instead sets $\theta_{i}$ so as to follow the boundary.

\section{Initialization and Loading}

The two propagation methods are initialized in different ways. In both cases, the information-bearing vesicles start out in a "loading zone", and their initial locations are selected at random and uniformly distributed within that region. In Brownian motion, at the start of the simulation, all vesicles are assumed to begin propagating simultaneously. Using active transport, the vesicles are assumed to be anchored to the loading zone until loaded on a microtubule, before propagating to the unloading zone. The initial position of the microtubules is selected at random and uniformly distributed within the entire region of propagation. The initial angle $\theta_{0}$ is selected uniformly at random from the range $[0,2 \pi]$, and microtubules are assumed to be initially unloaded unless their initial position 

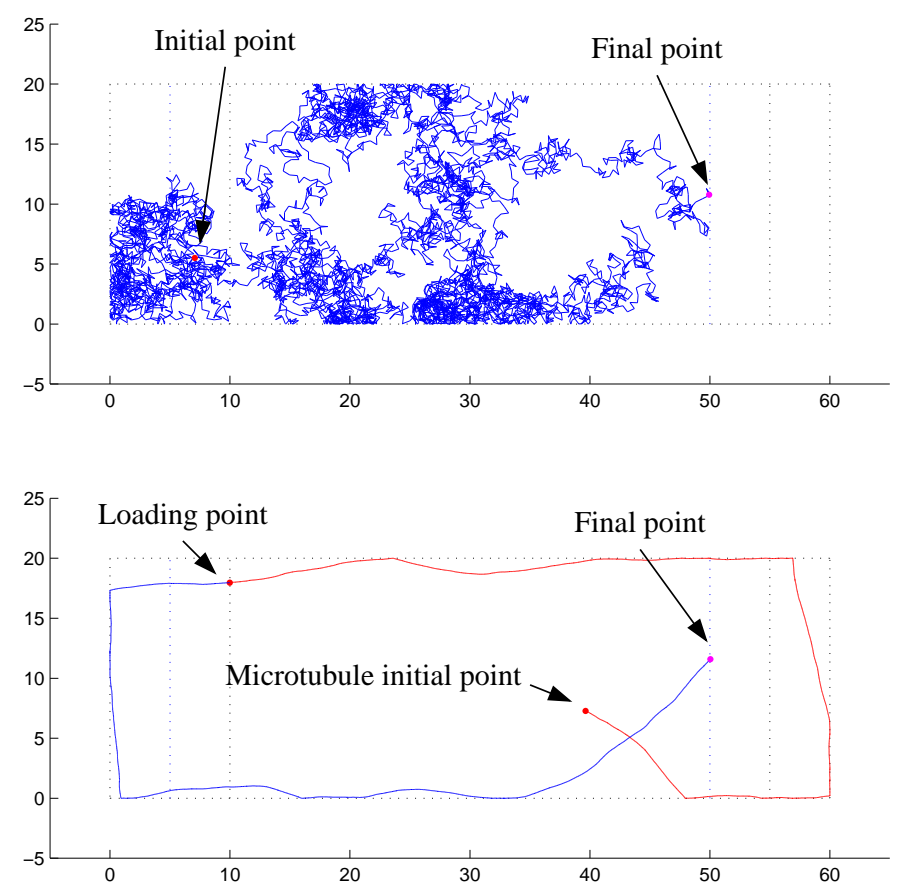

Fig. 2. Example simulated trajectories of Brownian motion (top) and active transport (bottom). Boundaries of the propagation environment are designated by dotted lines, and the loading and unloading zones are designated by dotted strips (loading on the left, unloading on the right). In the active transport trajectory, the red path indicates the unloaded motion of the microtubule.

is within the loading zone. When the microtubule arrives at a loading zone, for simplicity we assume that it loads exactly one vesicle. Experiments show that it is possible for microtubules to load multiple vesicles at once [13], and we will consider this scenario in future work.

Example simulated trajectories for both Brownian motion and active transport are given in Figure 2.

\section{INFORMATION TRANSMISSION}

Previous work has considered molecular communication either as a timing channel problem (i.e., where information is encoded in the times when molecules are released), or as a inscribed matter problem (i.e., where information is encoded by transmitting custom-made molecules, such as specific strands of DNA). A novel approach is taken in this paper: we consider information transmission as a mass transfer problem - in other words, a message is transmitted by moving a number of vesicles from the loading zone to the unloading zone.

In the simplest possible conception of this scheme, the vesicles themselves are not information-bearing, and a message is conveyed in the number of vesicles arranged on the loading zone. For example, if a maximum of three vesicles may be used, we may form messages two bits (i.e., $\log _{2} 4$ ): " 00 " for 0 vesicles, " 01 " for 1 vesicle, " 10 " for 2 vesicles, and " 11 " for 3 vesicles. However, this message is not perfectly conveyed to the receiver: given a time limit $T$ for the communication session, it is possible that some of the vesicles will not arrive at the unloading zone after $T$ has elapsed.

Let $x$ represent the random number of vesicles present at the loading zone, and let $y$ represent the number that arrive at the unloading zone once $T$ seconds have elapsed. Clearly, there exists some probability mass function $f(y \mid x)$ of the number of arrived vesicles given the number of transmitted vesicles. We are interested in the Shannon mutual information $I(X ; Y)$, given by

$$
I(X ; Y)=E\left[\log _{2} \frac{f(y \mid x)}{\sum_{x} f(y \mid x) f(x)}\right],
$$

where, in this example, $f(y \mid x)$ represents the probability of observing $y$ vesicles at the unloading zone, given that $x$ were released; $f(x)$ represents the probability of releasing $x$ vesicles at the loading zone; and $E[\cdot]$ represents expectation. The value of $I(X ; Y)$ represents the maximum rate at which data can be reliably sent over the link, measured in bits per time $T$.

The function $f(y \mid x)$ is obtained from our simulator. Whether Brownian motion or active transport is used, we assume that each vesicle's motion is independent from any other vesicle. In Brownian motion, it is sufficient to determine the probability that a given vesicle will arrive at the unloading zone within time $T$. Letting $p_{a}$ represent this probability, the function $f(y \mid x)$ has the binomial distribution, given by

$$
f(y \mid x)=\left\{\begin{array}{cc}
\left(\begin{array}{l}
x \\
y
\end{array}\right) p_{a}^{y}\left(1-p_{a}\right)^{x-y}, & 0 \leq y \leq x \\
0, & \text { otherwise }
\end{array}\right.
$$

Thus, $p_{a}$ is found by simulating many trials of vesicle motion over $T$ seconds, and counting the fraction that arrive.

For active transport, we obtain $f(y \mid x)$ directly by simulating many trials of a single microtubule over $T$ seconds, and determining how many vesicles are carried from the loading zone to the unloading zone in each trial. Thus, for a single microtubule, the function $f(y \mid x)$ is formed by taking the histogram of the trials.

If multiple microtubules are used, the probability mass function can be found from the histogram for a single microtubule, as follows. Let $y_{i}$ represent the number of microtubules transported by the $i$ th microtubule (for $i=1,2, \ldots, k$ ); clearly, $y=\sum_{i=1}^{k} y_{i}$. Also let $f(y \mid x ; k)$ represent the probability mass function for $k$ microtubles; then each $y_{i}$ is an independent and identically distributed random variable, with probability mass function $f(y \mid x ; 1)$, given by the histogram described above. Finally, we have

$$
f(y \mid x ; k)=\underbrace{f(y \mid x ; 1) \otimes f(y \mid x ; 1) \otimes \ldots \otimes f(y \mid x ; 1)}_{k \text { times }},
$$

where $\otimes$ represents convolution, which follows from a wellknown theorem for the sum of independent random variables.

\section{RESUlts}

In Figure 3, we depict a histogram obtained by simulating the motion of a single microtubule over a time $T=66.67 \mathrm{~min}$ (i.e., $4000 \mathrm{~s}$, or $66 \mathrm{~min} 40 \mathrm{~s}$ ); this is used as the probability density funciton $f(y \mid x ; 1)$ discussed in the previous section. 


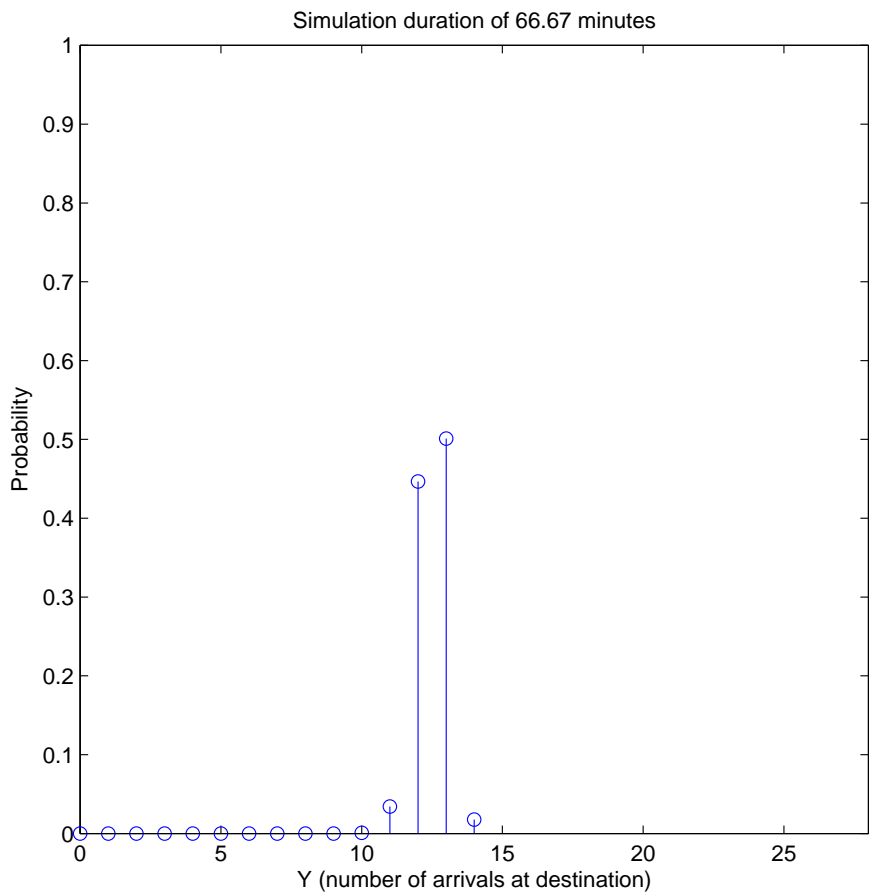

Fig. 3. Histogram of the number of vesicles transported by a single microtubule in time $T=66.67 \mathrm{~min}$.

This figure is typical of the results obtained for $f(y \mid x ; 1)$, with the number of arrivals concentrated very close to the mean. It is interesting to note that these distributions have low variance.

In Figure 4, we compare the achievable information rate using either Brownian motion or active transport as a function of the available number of vesicles; we assume that the transmitter's strategy is to use a uniform distribution over whatever number of vesicles is available, thus maximizing the transmitter's entropy. Even for a single microtubule, we see a large advantage using motors when the number of available vesicles (i.e., $x_{\max }$ ) is small. We conjecture that this is because the variance of the number of arrivals is relatively high in Brownian motion, whereas active transport has lower variance (i.e., given the number of transmitted vesicles, one can more reliably predict the number of vesicles received using microtubules, rather than using Brownian motion). However, information transfer using motors reaches a maximum, because the transfer of mass is limited by the number of available microtubules. This is suggested by the result in Figure 3, where there is zero probability of 15 or more vesicles arriving. Meanwhile, as expected, no such maximum exists for Brownian motion, since all the vesicles can propagate at the same time. Thus, we have two modes of operation: for small $x_{\max }$, active transport has the advantage; while as $x_{\max } \rightarrow \infty$, Brownian motion has the advantage: in the figure, Brownian motion has higher mutual information when $x_{\max } \geq 24$, compared to the peak of active transport. This is true for any setting of the system parameters (such as $T$ or the number of microtubules), since $I(X ; Y)$ for Brownian motion increases

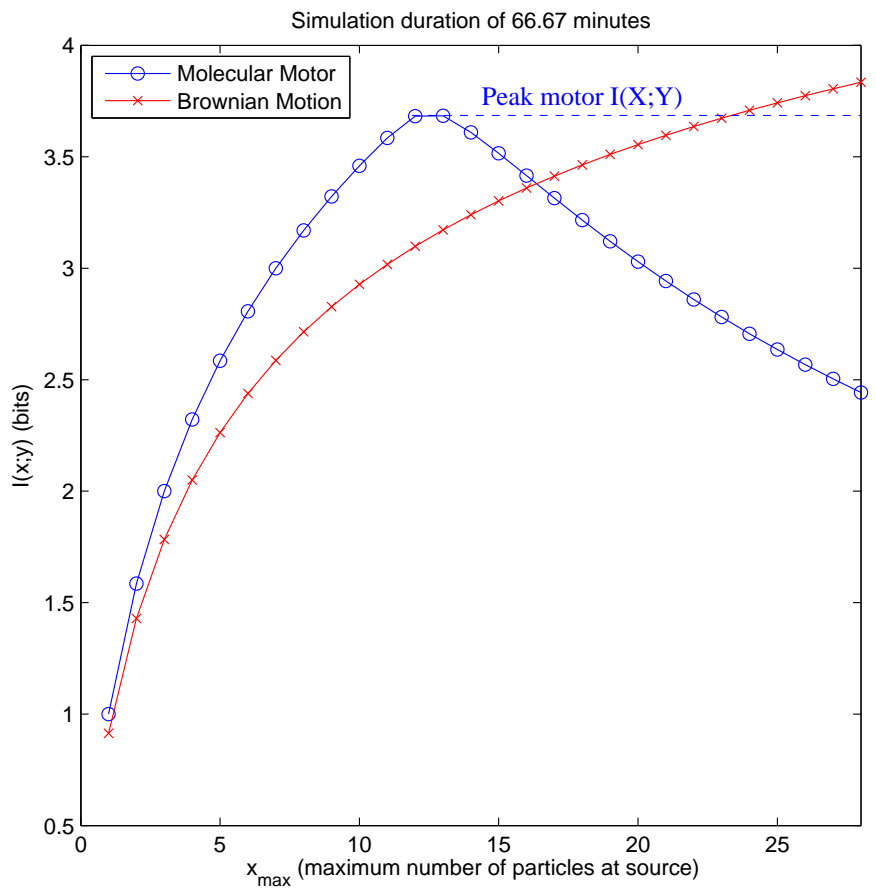

Fig. 4. Mutual information versus $x_{\max }$, where $T=66.67 \mathrm{~min}$. The distribution $f(x)$ is uniform over $\left\{0,1, \ldots, x_{\max }\right\}$. For motors, a maximum occurs because the number of vesicles that can be transported is limited.

with $x_{\max }$, but is generally bounded in active transport.

In Figure 5, we present results illustrating the effect of additional microtubules. As expected, the mutual information increases significantly as the number of microtubules increases, since it is possible to transport additional vesicles. However, the increase in mutual information is only logarithmic in the number of microtubules, so greater relative improvement is expected for small numbers of microtubules. In Figure 6, we compare multiple microtubules to Brownian motion, with respect to the entropy of the source; thus, this is a measure of the efficiency of information transfer. Since $f(x)$ is uniform over $\left\{0,1, \ldots, x_{\max }\right\}$, the value of the source entropy is $\log _{2}\left(1+x_{\max }\right)$. We see that the peak efficiencies for multiple microtubules are consistently close to 1 , whereas those for Brownian motion are much lower. Nonetheless, Brownian motion continues to dominate for large $x_{\max }$.

\section{CONCLUSION}

In this paper, we performed the first direct comparison of information transfer between Brownian motion and molecular motors. From our results, we may conclude that there are two modes of operation: if vesicles are scarce, molecular motors are superior, because of the lower variance in the propagation time; but if vesicles are plentiful, then Brownian motion is superior, because all of the vesicles are able to propagate at once. Our work leaves many interesting open questions for future research. For example, the effect of Brownian motion with drift, the effects of information-bearing vesicles, and the 


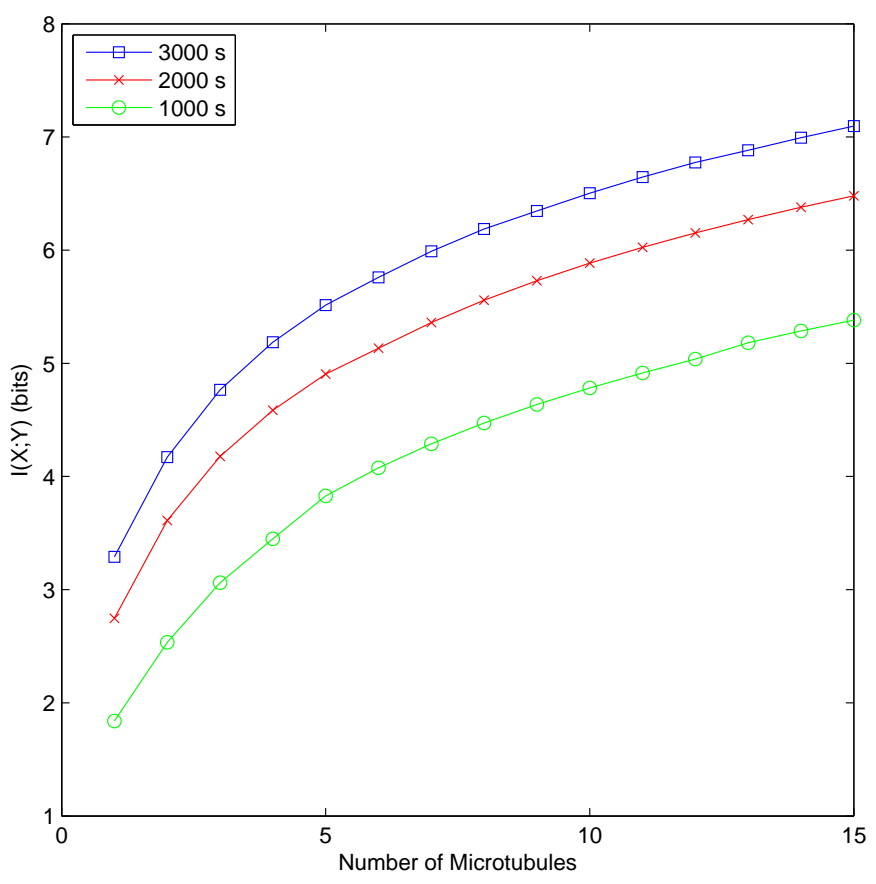

Fig. 5. Mutual information versus number of microtubules. In each case, the distribution $f(x)$ is uniform over $\left\{0,1, \ldots, x_{\max }\right\}$, and $x_{\max }$ is chosen to maximize mutual information.

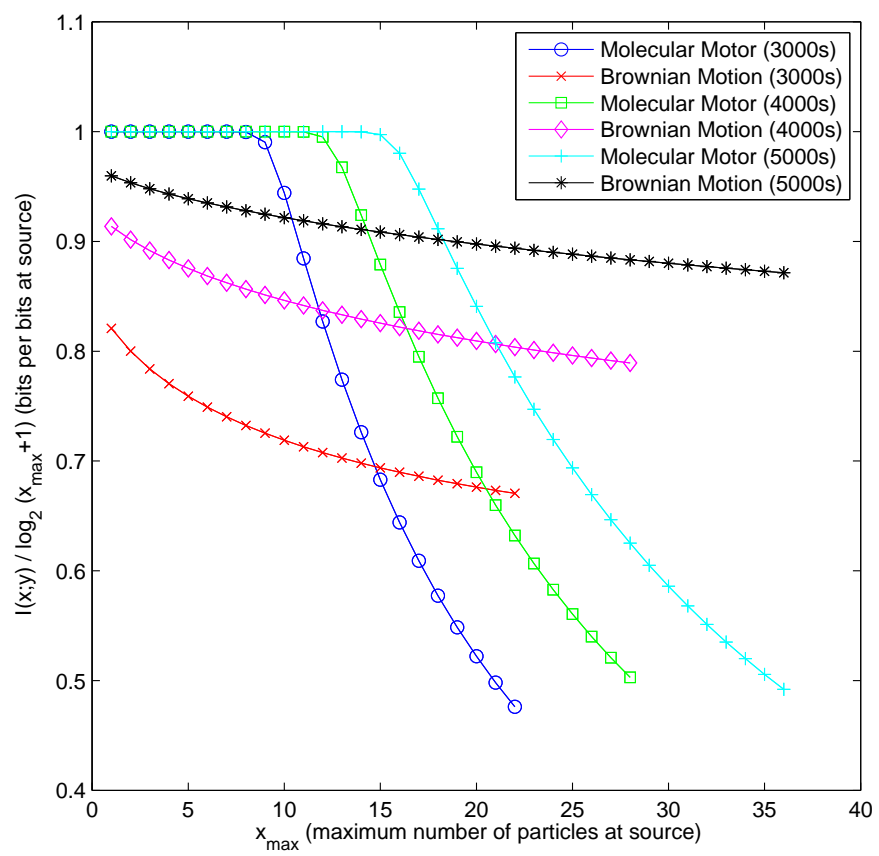

Fig. 6. A plot comparing mutual information with multiple microtubules to Brownian motion, plotted with respect to the source entropy $\log _{2}\left(1+x_{\max }\right)$. effect of multiple vesicle loadings on a single microtubule may be considered.

\section{REFERENCES}

[1] S. Hiyama et al., "Molecular communication," in Proc. 2005 NSTI Nanotechnology Conference, pp. 391-394, 2005.

[2] T. Nakano et al., "Molecular communication for nanomachines using intercellular calcium signalling," in Proc. 5th IEEE Conference on Nanotechnology, pp. 478-481, 2005.

[3] T. Nakano et al., "Molecular communication through gap junction channels: System design, experiments and modeling," in Proc. 2nd International Conference on Bio-Inspired Models of Network, Information, and Computing Systems, Budapest, Hungary, 2007.

[4] A. Enomoto et al., "A molecular communication system using a network of cytoskeletal filaments," in Proc. 2006 NSTI Nanotechnology Conference, pp. 725-728, 2006.

[5] S. Hiyama, Y. Moritani, and T. Suda, "A biochemically engineered molecular communication system," in Proc. 3rd International Conference on Nano-Networks, Boston, MA, USA, 2008.

[6] S. Hiyama and Y. Moritani, "Molecular communication: Harnessing biochemical materials to engineer biomimetic communication systems," Nano Communicaiton Networks, vol. 1, pp. 20-30, 2010.

[7] A. W. Eckford, "Nanoscale communication with Brownian motion," in Proc. Conf. on Information Sciences and Systems, Baltimore, MD, pp. 160-165, 2007.

[8] B. Atakan and O. Akan, "An information theoretical approach for molecular communication," in Proc. 2nd Intl. Conf. on Bio-Inspired Models of Network, Information, and Computing Systems, Budapest, Hungary, 2007.

[9] M. J. Moore, T. Suda, and K. Oiwa, "Molecular communication: Modeling noise effects on information rate, IEEE Transactions on Nanobioscience, vol. 8, pp. 169-179, Jun. 2009.

[10] A. W. Eckford, "Timing information rates for active transport molecular communication," in Proc. 4th Intl. Conf. on Nano-Networks, Luzern, Switzerland, pp. 24-28, 2009.

[11] R. Daw and J. Finkelstein (eds.), "Lab on a chip," Nature Insight, vol. 442, no. 7101, pp. 367-418, 2006.

[12] H. Hess and V. Vogel, "Molecular shuttles based on motor proteins: active transport in synthetic environments," Reviews in Molecular Biotechnology, vol. 82, pp. 67-85, 2001.

[13] S. Hiyama et al., "Biomolecular-motor-based nano- or microscale particle translocations on DNA microarrays," Nano Letters, vol. 9, no. 6, pp. 2407-2413, Jun. 2009.

[14] J. Berthier, Microfluidics for Biotechnology, Boston: Artech House, 2006.

[15] T. Nitta, A. Tanahashi, M. Hirano, and H. Hess, "Simulating molecular shuttle movements: Towards computer-aided design of nanoscale transport systems," Lab on a Chip, vol. 6, pp. 881-885, 2006. 